\title{
Quasistatic domains in planar nematic liquid crystals around the dielectric inversion point
}

\author{
V. G. Chigrinov, ${ }^{1}$ A. Sparavigna, ${ }^{2, *}$ and A. Strigazzi ${ }^{2, *}$ \\ ${ }^{1}$ Organic Intermediates \& Dyes Institute, Bolshaya Sadovaya 1-4, R-103787, Moscow, Russia \\ ${ }^{2}$ Dipartimento di Fisica, Politecnico di Torino, Corso Duca degli Abruzzi 24, I-10129, Torino, Italy
}

(Received 21 September 1995)

\begin{abstract}
A simple viscoelastic approach is proposed to describe the periodic patterns, characterized by static walls and splay-bend distortion, which appear in samples of nematic liquid crystals having dielectric anisotropy $\varepsilon_{a}$ dependent on the frequency. The modulated structure, resulting from a steady velocity field $\mathbf{v}$ coupled with a steady director field $\mathbf{n}$, is achieved when an electric field is applied normally to the plates of a planar unidirectional nematic cell. Such a kind of quasistatic domain is theoretically investigated not only in the frequency region, where the usual aperiodic Fréedericksz effect becomes unfavorable, $\operatorname{Re}\left(\varepsilon_{a}\right)$ still being positive, but also where $\operatorname{Re}\left(\varepsilon_{a}\right)<0$, favoring in principle the initial orientation. Both previous situations are considered in the vicinity of the sign reversal point. The present model describes the dielectric loss near the reversal point in terms of the appearance of the corresponding effective space charge, which interacts with the effective electric field, causing a steady electrohydrodynamic motion of very small amplitude inside the nematic liquid crystal layer. As a result, a quasistatic tilted modulated structure emerges, with wave vector parallel to the initial planar orientation of the nematic cell.
\end{abstract}

PACS number(s): $61.30 .-\mathrm{v}$

\section{INTRODUCTION}

The electrically controlled Fréedericksz effect in nematic liquid crystals (NLCs) is known to occur due to the dielectric coupling between the nematic director $\mathbf{n}$ and an external field $\mathbf{E}$ applied to a NLC layer properly aligned, for instance, in a configuration without any distortion. In particular, let us consider a unidirectional planar $(P)$ cell, filled by a NLC with the real part of the dielectric anisotropy $\operatorname{Re}\left(\varepsilon_{a}\right)$, supposed to be positive at low frequency. The corresponding interaction energy density is given by $f_{E} \cong-\varepsilon_{0} \operatorname{Re}\left(\varepsilon_{a}\right) E^{2} \theta^{2} / 2$ for small director tilt angles $\theta(|\theta| \ll 1)$ from the initial $\mathrm{P}$ orientation, if $\mathbf{E}$ is normal to the cell plates [1]. Obviously $f_{E}$ goes to zero for low values of the real dielectric anisotropy $\operatorname{Re}\left(\varepsilon_{a}\right)>0$ and disappears when the NLC reaches the dielectric isotropic point $\operatorname{Re}\left(\varepsilon_{a}\right)=0$ : for instance, such a condition can be reached for several mixtures of NLC compounds, by increasing the angular frequency $\omega$ of the applied electric field $\mathbf{E}(\omega)$.

In fact, special interest should be paid indeed for both fundamental and practical reasons to those NLCs that exhibit a sign reversal of the real dielectric anisotropy $\operatorname{Re}\left(\varepsilon_{a}\right)$ with the angular frequency $\omega$ of the applied field $E=E_{0} \cos \omega t$. From an application point of view, it appears particularly interesting, when the sign inversion point $\omega=\omega_{i}$ of the dielectric anisotropy is in the $\mathrm{kHz}$ region. A periodic domain structure was observed earlier in planar NLC cells $[2,3]$, close to $\omega=\omega_{i}$, comprising a range where $\operatorname{Re}\left(\varepsilon_{a}\right)$ is given by

$$
\begin{aligned}
& \operatorname{Re}\left[\varepsilon_{a}\left(\omega<\omega_{i}\right)\right]>0, \\
& \operatorname{Re}\left[\varepsilon_{a}\left(\omega=\omega_{i}\right)\right]=0, \\
& \operatorname{Re}\left[\varepsilon_{a}\left(\omega>\omega_{i}\right)\right]<0
\end{aligned}
$$

\footnotetext{
*Authors to whom correspondence should be addressed.
}

and was considered as a pure electrohydrodynamic phenomenon $[4,5]$. The electrohydrodynamic model of Goossens [4] in fact gave an approximate explanation of the stripes appearance in a NLC layer in the presence of ions, in the framework of a one-dimensional approach.

Careful experiments have been performed [6,7], showing the appearance of quasistatic stripes in NLC cells obtained with mixtures of butyl-methoxyazoxybenzole and butylheptyloxyazoxybenzole, doped with cyanophenyl esters of benzoic acids. The quasistatic stripes are characterized by the splay-bend distortion only, where a steady director field $\mathbf{n}$ is coupled with a steady velocity field $\mathbf{v}$, providing static stripe walls.

In this case, we will prove that neither the parallel ionic conductivity $\sigma_{\|}$nor the ionic conductivity anisotropy $\sigma_{a}$ influences the instability threshold, whereas the threshold itself turns out to be strictly dependent on the ratio $\omega_{i} / \sigma_{\perp}, \sigma_{\perp}$ being the perpendicular conductivity, and on the Leslie's viscosity coefficients $\alpha_{2}$ and $\alpha_{5}$ [8]. We will treat these domains as due to a generalized periodic Fréedericksz transition [9-11] involving also steady hydrodynamics.

Figure 1 qualitatively shows the behavior of the external electric potential at the threshold $U_{c}$ as a function of the angular frequency $\omega$ of the applied electronic field $\mathbf{E}$ in the case of a NLC exhibiting $\varepsilon_{a}=\varepsilon_{a}(\omega)$ and filling a cell with initial $P$ orientation. Near the inversion frequency, where the Fréedericksz threshold diverges, there is a critical frequency $\omega_{p}<\omega_{i}$ such that the periodic domains arise and, as a consequence, the diagram shows the contemporary presence of three possible configurations, involving the unidirectional planar, aperiodic, and periodic alignments, respectively.

\section{THEORY}

The aim of the present paper is to give a simple explanation of the physical origin of the quasistatic domain structure not as a pure dielectric effect, like it happens in ideal insu- 


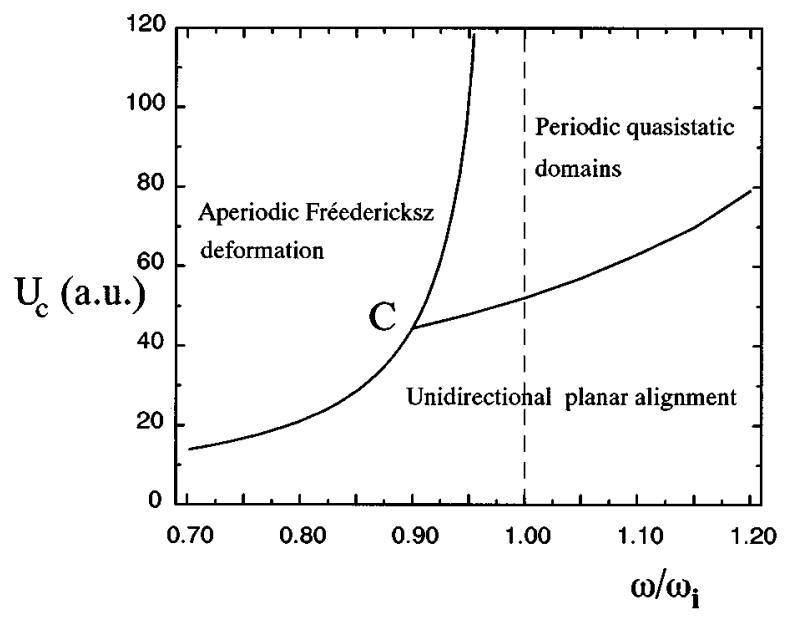

FIG. 1. Critical electric potential $U_{c}$ (in arbitrary units) for aperiodic and periodic splay-bend distortion, as a function of the reduced angular frequency $\omega / \omega_{i}$, where $\omega_{i}$ is the inversion point of the real permittivity anisotropy $\delta \varepsilon \equiv \varepsilon^{\prime}-\varepsilon_{\perp}$. When $\omega<\omega_{i}$, then $\delta \varepsilon>0$ and vice versa. The phase diagram shows, for NLC materials similar to the mixtures used in the experiment [6], the regions where the initially undistorted $P$ alignment, the aperiodic Freedericksz deformation, and the periodic quasistatic domains are stable, respectively. Note the presence of the tricritical point $C$ and, remarkably, the fact that the periodic steady deformation appears also for $\delta \varepsilon<0$, provided that the potential $U$ is high enough.

lating materials, but as a complex dielectric effect, resulting in a steady director reorientation, spatially periodic in the plane $(x, z)$, involving just a splay-bend distortion (see Fig. 2, where the $x$ axis characterizes the $P$ easy direction along both substrates and the $z$ axis is normal to the substrates themselves). The director field $\mathbf{n}$ couples with a steady molecular velocity field $\mathbf{v}$, allowing the domain walls to be fixed.

Such a periodic pattern is accompanied by a spatial regular induced potential distribution, providing an amplitude modulated internal contribution $\mathbf{E}_{I}$ to the effective electric field $\mathbf{E}_{e}=\mathbf{E}+\mathbf{E}_{I}$, which turns out to be nonlocal, but dependent on the already established deformation in the whole cell.

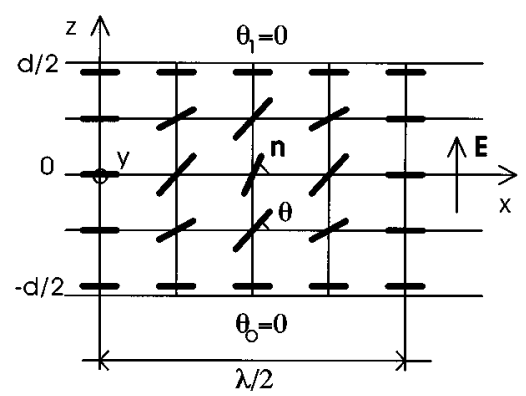

FIG. 2. Periodic splay-bend steady distortion in the $(x, z)$ plane for a NLC having parallel complex permittivity $\varepsilon_{\|}$dependent on the angular frequency $\omega$ of the applied electric field. The NLC director $\mathbf{n}$, initially in the unidirectional planar $(P)$ configuration along the $x$ axis, is kept aligned parallel to the $x$ axis at the substrate, due to the strong anchoring. The external electric field $\mathbf{E}$ is applied along the $z$ axis normal to the cell plates.
Let us consider the external electric field $\mathbf{E}$ with amplitude

$$
E(t)=E_{0} \cos \omega t=E_{0} \operatorname{Re}\left(e^{i \omega t}\right)
$$

as applied along the $z$ axis. The NLC filling the cell could be characterized by a Debye length either much greater than or of the same order as the layer thickness. Hence, in the first case it can only be considered as a perfect insulator, whereas in the second one its conductivity cannot be neglected. We will consider the more general case, taking into account the possible presence of space charges.

The nematic director $\mathbf{n}$ deviates to the small tilt angle $\theta$ from the initial $P$ orientation along the $x$ axis, belonging to the plane $(x, z)$ : this is the simplest deformation, providing no azimuthal variation. Hence the director field reads

$$
\mathbf{n} \sim(1,0, \theta) \quad \text { where }|\theta| \ll 1 \text {. }
$$

The dielectric displacement vector $\mathbf{D}$ is given by

$$
\frac{\mathbf{D}}{\varepsilon_{0}}=\varepsilon_{\perp} \mathbf{E}_{e}+\varepsilon_{a}\left(\mathbf{E}_{e} \cdot \mathbf{n}\right) \mathbf{n},
$$

where the complex permittivity anisotropy is $\varepsilon_{a} \equiv \varepsilon_{\|}-\varepsilon_{\perp}, \varepsilon_{\|}$ and $\varepsilon_{\perp}$ being the dielectric constants parallel and perpendicular to the NLC director, respectively. The parallel permittivity $\varepsilon_{\|}$varies with the angular frequency $\omega$ according to the Debye model:

$$
\begin{gathered}
\varepsilon_{\|}=\varepsilon^{\prime}-i \varepsilon^{\prime \prime}, \\
\varepsilon^{\prime}=\varepsilon_{\infty}+\frac{\varepsilon_{0}-\varepsilon_{\infty}}{1+\left(\omega / \omega_{D}\right)^{2}}, \\
\varepsilon^{\prime \prime}=\frac{\left(\varepsilon_{0}-\varepsilon_{\infty}\right) \omega / \omega_{D}}{1+\left(\omega / \omega_{D}\right)^{2}},
\end{gathered}
$$

where $\varepsilon_{0}=\varepsilon^{\prime}(\omega=0), \varepsilon_{\infty}=\varepsilon^{\prime}(\omega \rightarrow \infty)$, and $\omega_{D}$ is Debye frequency. At the same time the normal permittivity $\varepsilon_{\perp}$ is supposed to be real and almost constant in this frequency region.

At the angular frequency $\omega=\omega_{i}$ of the dielectric sign reversal, the uniform Fréedericksz transition cannot take place, since the corresponding contribution $f_{E}$ to the nematic freeenergy density vanishes. In fact, in the case of uniform Fréedericksz transition, the NLC mixture behaves as it would for a perfect dielectric material, since only the real part of the dielectric tensor matters: hence just the real part of the dielectric anisotropy $\delta \varepsilon \equiv \varepsilon^{\prime}-\varepsilon_{\perp}$ plays a role. Furthermore, the free-energy density due to the dielectric coupling reads

$$
f_{E}=-\frac{\mathbf{D} \cdot \mathbf{E}}{2}=-\frac{D_{z} E}{2},
$$

where $\mathbf{E}$ is the external field, since due to the absence of any modulation, the internal contribution $\mathbf{E}_{I}$ may be neglected [1]. Also $D_{z}$ turns out to be the $z$ component of the external electric displacement

$$
\frac{D_{z}}{\varepsilon_{0}}=\operatorname{Re}\left(\varepsilon_{\perp} E+\varepsilon_{a} E \theta^{2}\right)
$$


and the time average (in angular brackets \langle\rangle ) of the electric free-energy density is written

$$
\begin{aligned}
\left\langle f_{E}\right\rangle=-\left\langle\frac{D_{z} E}{2}\right\rangle= & -\frac{\varepsilon_{0}}{2 T}\left[\int_{0}^{T} \operatorname{Re}\left(\varepsilon_{\perp} E\right) E d t\right. \\
& \left.+\theta^{2} \int_{0}^{T} \operatorname{Re}\left(\varepsilon_{a} E\right) E d t\right]
\end{aligned}
$$

where $T=2 \pi / \omega$ is the period of the applied field. Note that $\theta$ is time independent, since only quasistatic period patterns are investigated: as previously stated, $\mathbf{n}$ is a steady field. The first term in (8) does not depend on the director orientation and could be omitted, i.e., the Fréedericksz phenomenon is affected only by the second term. The latter one, according to Eqs. (2) and (5), is written as

$$
\begin{aligned}
-\frac{\varepsilon_{0} \theta^{2}}{2 T} \int_{0}^{T} \operatorname{Re}\left(\varepsilon_{a} E\right) E d t= & -\frac{\varepsilon_{0} \theta^{2}\left|\varepsilon_{a}\right| E_{0}^{2}}{2 T} \\
& \times \int_{0}^{T} \cos (\omega t+\alpha) \cos \omega t d t,
\end{aligned}
$$

where $\alpha=\arg \left(\varepsilon_{a}\right)=-\arctan \left[\varepsilon^{\prime \prime} /\left(\varepsilon^{\prime}-\varepsilon_{\perp}\right)\right]$. At the point of dielectric isotropy, we obtain $\varepsilon^{\prime}=\varepsilon_{\perp}$ and $|\alpha|=\pi / 2$, which means that the second term is zero, i.e., the uniform aperiodic Fréedericksz effect cannot take place (see Fig. 1).

Let us suppose now that the Freedericksz transition takes place in the form of a modulated structure. Hence, as we mentioned above, the effective electric field $\mathbf{E}_{e}$ is the superposition of the applied electric field $\mathbf{E}(t)$ and of the small internal field $\mathbf{E}_{I}(x, z, t)$. Such a nonlocal contribution cannot be neglected, as in the usual situations. In fact, when the periodic stripes exist, also the internal field must present a spatially modulated structure $\mathbf{E}_{I}(x, z, t)=-\nabla \psi(x, z) E(t)$, which influences the periodic pattern in its turn, as a feedback effect. Note that the characteristic length $\psi(x, z)$ can be defined as the reduced potential of the internal electric field, taking into account locally the effect of the nonlocal distortion in the whole cell. In this case the effective electric field becomes dependent on both coordinates $(x, z)$ too and reads

$$
\mathbf{E}_{e}=\left(-\frac{\partial \psi}{\partial x}, 0,1-\frac{\partial \psi}{\partial z}\right) E
$$

Since $\mathbf{E}_{I}$ is small as compared with $\mathbf{E}$, then also $|\nabla \psi|$ must be small, such as

$$
\left\{\left|\frac{\partial \psi}{\partial x}\right|,\left|\frac{\partial \psi}{\partial z}\right|\right\} \ll 1 .
$$

The variations of the electric-field-reduced potential $\psi(x, z)$ are coupled with those of the director tilt angle $\theta$ by the Maxwell equation $\operatorname{div}\left(\mathbf{D} / \varepsilon_{0}\right)=\operatorname{div}\left[\varepsilon_{\perp} \mathbf{E}_{e}+\varepsilon_{a}\left(\mathbf{E}_{e} \cdot \mathbf{n}\right) \mathbf{n}\right]=Q / \varepsilon_{0}$, providing the local Gauss law in the mesogenic material, in the form

$$
\varepsilon_{\|} E \frac{\partial^{2} \psi}{\partial x^{2}}+\varepsilon_{\perp} E \frac{\partial^{2} \psi}{\partial z^{2}}-\varepsilon_{a} E \frac{\partial \theta}{\partial x}+\frac{Q}{\varepsilon_{0}}=0,
$$

where the spatial charge density $Q$ is a priori due to the dielectric loss and to the ionic conductivity.

Since the external electric field $E(t)$ is harmonic according to Eq. (2), consequently, neglecting the contribution of the higher-order harmonics, the effective spatial charge density $Q$ is supposed to obey to the harmonic law with a phase shift with respect to the imposed field $\mathbf{E}$ :

$$
Q=Q_{c}(x, z) \cos \omega t+Q_{s}(x, z) \sin \omega t
$$

The effective spatial charge density $Q$ interacts with the effective electric field $\mathbf{E}_{e}$, exerting a drag force $Q \mathbf{E}_{e}$ per volume unit in the NLC cell, thus causing an electrohydrodynamic flow. Keeping only the zeroth-order term in the interaction, the drag force can be written as $\sim Q \mathbf{E}$, where $\mathbf{E}$ is the external field only. Due to the symmetry, the velocity field $\mathbf{v}$ is given by

$$
\mathbf{v}=\left(v_{x}, 0, v_{z}\right)
$$

Let us stress once more that all the small parameters $\xi \equiv\left(\theta, \psi, Q_{c}, Q_{s}, v_{x}, v_{z}\right)$ do not depend on the $y$ coordinate, since the present model is concerned only with domain walls oriented along such a direction. For this reason, in Eq. (14) $v_{y}=0$ too. In fact, the experiment shows that a linearly polarized light beam along the $x$ direction gives a modulated pattern, whereas a light beam polarized along the $y$ direction does not give any diffraction picture.

We suppose that the $(x, z)$ dependence of the abovementioned unknown parameters $\xi$ could be written as $\xi=\xi_{0} \exp \left(i q_{x} x+i q_{z} z\right)$ [12], where the subset $\xi_{0} \equiv\left[\theta, \psi, v_{x}, v_{z}\right]$ generally can be time dependent, $q_{x}, q_{z}$ being the wave vectors of the domains along the $x$ and $z$ directions, respectively. The linear electrohydrodynamic model describing the problem includes, together with Gauss's law (12), also (i) the Maxwell equation connected with the effective current density

$$
\frac{d Q}{d t}+\operatorname{div} \mathbf{j}=0
$$

where $\mathbf{j}=\widetilde{\sigma} \mathbf{E}_{e}$ and $\widetilde{\sigma} \equiv\left\{\sigma_{i k}\right\}=\left\{\sigma_{\perp} \delta_{i k}+\sigma_{a} n_{i} n_{k}\right\}$ is the complex conductivity tensor; (ii) the Navier-Stokes equations, i.e., the Newton law connecting the particle acceleration components $d v_{i} / d t$ in the anisotropic liquid with the internal pressure $P$, the electrical drag force $Q E_{i}$, and the viscous stresses $\partial \Sigma_{i j} / \partial x_{j}$,

$$
\rho \frac{d v_{i}}{d t}=-\frac{\partial P}{\partial x_{i}}+Q E_{i}+\frac{\partial \Sigma_{i j}}{\partial x_{j}}
$$

where $\rho$ is the mass density and $\Sigma_{i j}$ is the Leslie-Ericksen viscosity tensor [8], expanded according to Rivlin's rule [13-15] in terms of the director $\mathbf{n}$, of the emisymmetric velocity gradient $A_{i j}$, and of the director substantial derivative $\mathbf{N}$, where the linear combination coefficients are [1] Leslie's viscosities $\alpha_{i}(i=1, \ldots, 6)$, 


$$
\begin{gathered}
\sum_{i j}=\alpha_{1} n_{i} n_{k} A_{k m} n_{n} n_{m}+\alpha_{2} n_{i} N_{j}+\alpha_{3} n_{j} N_{i}+\alpha_{4} A_{i j} \\
+\alpha_{5} n_{i} n_{k} A_{k j}+\alpha_{6} A_{i k} n_{k} n_{j}, \\
A_{i k}=\frac{1}{2}\left(\frac{\partial v_{i}}{\partial x_{k}}+\frac{\partial v_{k}}{\partial x_{i}}\right) \\
\mathbf{N}=\frac{d \mathbf{n}}{d t}+\frac{1}{2}[\mathbf{n} \times \text { curlv }] ;
\end{gathered}
$$

(iii) the continuity equation for incompressible liquids

$$
\operatorname{div} \mathbf{v}=0
$$

which describes $v_{x}$ as dependent on $v_{z}$; and (iv)the torqueequation for NLC director rotation under the applied field

$$
\mathbf{n} \times \mathbf{h}=\Gamma=\mathbf{n} \times\left\{\gamma_{1} \mathbf{N}+\gamma_{2} \widetilde{A} \mathbf{n}\right\},
$$

where $\mathbf{h}=-(\delta f / \delta \mathbf{n})$ is the functional derivatives of the NLC free-energy density $f, \gamma_{1}$ and $\gamma_{2}$ are defined as $\gamma_{1} \equiv \alpha_{3}-\alpha_{2}$ and $\gamma_{2} \equiv \alpha_{3}+\alpha_{2}$, and $\widetilde{A} \equiv\left\{A_{i j}\right\}$.

The final set of the linear electrohydrodynamic equations in the parameters $\left[\zeta_{0} \equiv(\partial \theta / \partial x)_{0}, \psi_{0}, Q_{s}^{0}, Q_{c}^{0}, v_{0} \equiv v_{z 0}\right]$ may be derived from Eqs. (12), (15), (16), and (19) as [12,15]

$$
\begin{aligned}
& {\left[\left(\varepsilon^{\prime} q_{x}^{2}+\varepsilon_{\perp} q_{z}^{2}\right) \cos \tau+\varepsilon^{\prime \prime} q_{x}^{2} \sin \tau\right] E_{0} \psi_{0}} \\
& +\left(\delta \varepsilon \cos \tau+\varepsilon^{\prime \prime} \sin \tau\right) \zeta_{0} E_{0}=\left(Q_{c}^{0} \cos \tau+Q_{s}^{0} \sin \tau\right) / \varepsilon_{0} \\
& \left(-Q_{c}^{0} \omega \sin \tau+Q_{s}^{0} \omega \cos \tau\right)+\left[\left(\sigma^{\prime} q_{x}^{2}+\sigma_{\perp} q_{z}^{2}\right) \cos \tau\right. \\
& \left.+\sigma^{\prime \prime} q_{x}^{2} \sin \tau\right] E_{0} \psi_{0}+\left(\delta \sigma^{\prime} \cos \tau+\sigma^{\prime \prime} \sin \tau\right) \zeta_{0} E_{0}=0 \\
& \rho \omega\left(q_{x}^{2}+q_{z}^{2}\right) \frac{d v_{0}}{d \tau}+\left(\eta_{1} q_{x}^{4}+\eta_{12} q_{x}^{2} q_{z}^{2}+\eta_{2} q_{z}^{4}\right) v_{0} \\
& \quad+\left(\alpha_{3} q_{z}^{2}-\alpha_{2} q_{x}^{2}\right) \frac{d \zeta_{0}}{d \tau} \\
& \quad-q_{x}^{2} E_{0}\left(Q_{c}^{0} \cos ^{2} \tau+Q_{s}^{0} \cos \tau \sin \tau\right)=0 \\
& \gamma_{1} \omega \frac{d \zeta_{0}}{d \tau}+\zeta_{0}\left[K_{33} q_{x}^{2}+K_{11} q_{z}^{2}-\varepsilon_{0} E_{0}^{2}\left(\delta \varepsilon \cos ^{2} \tau\right.\right. \\
& \left.\left.+\varepsilon^{\prime \prime} \sin \tau \cos \tau\right)\right]-q_{x}^{2} \varepsilon_{0} E_{0}^{2} \psi_{0}\left(\delta \varepsilon \cos ^{2} \tau+\varepsilon^{\prime \prime} \sin \tau \cos \tau\right) \\
& +\left(\alpha_{3} q_{z}^{2}-\alpha_{2} q_{x}^{2}\right) v_{0}=0
\end{aligned}
$$

where $\tau \equiv \omega t$ is the reduced time, $\eta_{1}, \eta_{12}, \eta_{2}$ are the combinations of Leslie's viscosities [1,8]

$$
\begin{gathered}
\eta_{1}=\frac{1}{2}\left(\alpha_{4}+\alpha_{5}-\alpha_{2}\right), \\
\eta_{12}=\alpha_{1}+\alpha_{4}+\frac{1}{2}\left(\alpha_{5}+\alpha_{6}+\alpha_{3}-\alpha_{2}\right), \\
\eta_{2}=\frac{1}{2}\left(\alpha_{3}+\alpha_{4}+\alpha_{6}\right),
\end{gathered}
$$

and $K_{11}, K_{33}$ are splay and bend elastic moduli. We stress the fact that the component $v_{x}$ does not appear in the system (20) because it is related to $v_{z}$ by the continuity equation for incompressible fluid (18). Moreover, when writing system
(20), the complex nature of the permittivity and of the electric field has been taken into account, according to the relations [see Eq. (2)]

$$
\begin{aligned}
& \varepsilon_{\|} E=\operatorname{Re}\left[\left(\varepsilon^{\prime}-i \varepsilon^{\prime \prime}\right) E_{0}(\cos \tau+i \sin \tau)\right] \\
&=E_{0}\left(\varepsilon^{\prime} \cos \tau+\varepsilon^{\prime \prime} \sin \tau\right), \\
& \varepsilon_{a} E=\operatorname{Re}\left[\left(\delta \varepsilon-i \varepsilon^{\prime \prime}\right) E_{0}(\cos \tau+i \sin \tau)\right] \\
&=E_{0}\left(\delta \varepsilon \cos \tau+\varepsilon^{\prime \prime} \sin \tau\right), \\
& \varepsilon_{a}=\delta \varepsilon-i \varepsilon^{\prime \prime} .
\end{aligned}
$$

Similar relations were also considered for the relevant NLC conductivities, i.e., for the complex parallel conductivity $\sigma_{\|}=\sigma^{\prime}-i \sigma^{\prime \prime}$ and the perpendicular conductivity $\sigma_{\perp}$, as taken with respect to the NLC director. Note that $\sigma_{\perp}$ turns out to be real. Let us suppose that the system (20) has steady solutions $\zeta_{0}, \psi_{0}$, and $v_{0}$, i.e.,

$$
\frac{d \zeta_{0}}{d \tau}=\frac{d \psi_{0}}{d \tau}=\frac{d v_{0}}{d \tau}=0
$$

To evaluate the threshold of the domain structure around the inversion point $\omega=\omega_{i}$, where $\operatorname{Re}\left(\varepsilon_{a}\right)=\delta \varepsilon=0$, it is enough to compare the coefficients of the same order in $\cos \tau$ and $\sin \tau$ in the first two equations in the system (20).

Concerning the second two equations, it is possible either to average them over one period in time or to neglect the second harmonic contribution. The actual existence of the higher-order terms in $\cos (2 \tau)$ and $\sin (2 \tau)$ introduces only corrections of the order of

$$
\frac{\bar{K} q^{2}}{\gamma_{1} \omega} \ll 1, \quad \frac{\bar{\alpha} q^{2}}{\rho \omega} \ll 1,
$$

where $\bar{K}, \bar{\alpha}$ are averaged values of elastic constants and viscosities, respectively (see Ref. [12], p. 335). Such terms can be neglected in the present case, which comprises sufficiently large frequencies, around the sign inversion point.

For the sake of simplicity, let us assume the hypothesis of one elastic constant and let us consider the weighted average of the viscosity

$$
\begin{gathered}
K_{11}=K_{33} \equiv K, \\
\eta_{1} q_{x}^{4}+\eta_{12} q_{x}^{2} q_{z}^{2}+\eta_{2} q_{z}^{4} \sim \bar{\eta}\left(q_{x}^{2}+q_{z}^{2}\right)^{2} .
\end{gathered}
$$

Then the system (20) reads, setting $s \equiv \sigma^{\prime} / \sigma_{\perp}$ :

$$
v_{0}=\frac{q_{x}^{2} Q_{c}^{0} E_{0}}{2 \bar{\eta}\left(q_{x}^{2}+q_{z}^{2}\right)^{2}},
$$

$$
\begin{gathered}
\zeta_{0} K\left(q_{x}^{2}+q_{z}^{2}\right)-\frac{\varepsilon_{0} \delta \varepsilon E_{0}^{2}}{2}\left(\psi_{0} q_{x}^{2}+\zeta_{0}\right)+\left(\alpha_{3} q_{z}^{2}-\alpha_{2} q_{x}^{2}\right) v_{0}=0, \\
Q_{c}^{0} / \varepsilon_{0}=\left(q_{x}^{2} \varepsilon^{\prime}+\varepsilon_{\perp} q_{z}^{2}\right) E_{0} \psi_{0}+\delta \varepsilon E_{0} \zeta_{0}, \\
Q_{s}^{0} / \varepsilon_{0}=\varepsilon^{\prime \prime} E_{0}\left(\psi_{0} q_{x}^{2}+\zeta_{0}\right), \\
\omega Q_{s}^{0}+\sigma_{\perp}\left(s q_{x}^{2}+q_{z}^{2}\right) \psi_{0} E_{0}=0, \\
\omega Q_{c}^{0}=\sigma^{\prime \prime} E_{0}\left(q_{x}^{2} \psi_{0}+\zeta_{0}\right)+\delta \sigma E_{0} \zeta_{0} .
\end{gathered}
$$


It is worth noting that the system (26) allows us to determine the five parameters $\xi_{0}$ and the imaginary part of the conductivity $\sigma^{\prime \prime}$, which by definition gives an increase of the free energy embedded in the dielectric material. In particular, the last four equations of the system (26) enable us to express $\sigma^{\prime \prime}$ in terms of the dielectric loss $\varepsilon^{\prime \prime}$ :

$$
\begin{aligned}
\sigma^{\prime \prime}= & \varepsilon_{0} \varepsilon^{\prime \prime} \omega\left\{\delta \sigma \zeta_{0}-\varepsilon_{0} \omega\left[\left(\varepsilon^{\prime} q_{x}^{2}+\varepsilon_{\perp} q_{z}^{2}\right) \psi_{0}+\delta \varepsilon \zeta_{0}\right]\right\} \\
& \times\left[\sigma_{\perp}\left(s q_{x}^{2}+q_{z}^{2}\right) \psi_{0}\right]^{-1}
\end{aligned}
$$

which, for $\delta \sigma=0$, is simply written

$$
\sigma^{\prime \prime}=\varepsilon^{\prime \prime} \varepsilon_{0} \omega\left(\frac{\delta \varepsilon}{\varepsilon^{\prime \prime}}-\frac{\varepsilon_{\perp} \varepsilon_{0} \omega}{\sigma_{\perp}}\right)
$$

Limiting ourselves to the hypothesis of conductivity isotropy $\delta \sigma=0$ and considering Eq. (27'), the first five equations of the system (26) are linearly independent of the five parameters $\xi_{0} \equiv\left(\zeta_{0}, \psi_{0}, Q_{c}^{0}, Q_{s}^{0}, v_{0}\right)$. The condition of nontriviality of these parameters provides the dispersion relation between the applied field amplitude $E_{0}$ and the instability wave vectors $q_{x}, q_{z}$,

$$
\varepsilon_{0} E_{0}^{2}=\frac{\left.2 K \bar{\eta}\left(q^{2}+1\right)^{3}((\mathscr{B}+1)) q^{2}+1\right)}{q^{2}\left(q^{2}+1\right)\left(\alpha_{3}-\alpha_{2} q^{2}\right)\left[(\mathscr{B}+1) \varepsilon_{\perp}-\varepsilon^{\prime}\right]-\delta \varepsilon \bar{\eta}\left(q^{2}+1\right)^{3}} q_{z}^{2},
$$

where

$$
\mathscr{B} \equiv \frac{\varepsilon_{0} \varepsilon^{\prime \prime} \omega}{\sigma_{\perp}}
$$

and the in-plane wave vector, reduced with respect to the out-of-plane wave vector, is $q \equiv q_{x} / q_{z}$, as defined. In the general case, when $\delta \sigma \neq 0$, the potential $U=\pi E / q_{z}$ is obtained as

$$
\varepsilon_{0} \frac{U_{0}^{2}(q)}{K_{11}}=\frac{2 \pi^{2}\left(K_{33} / K_{11} q^{2}+1\right)\left(\eta_{1} q^{4}+\eta_{12} q^{2}+\eta_{2}\right)\left[(\mathscr{B}+s) q^{2}+1\right]}{\left(q^{2}+1\right)\left\{q^{2}\left[(\mathscr{B}+s) \varepsilon_{\perp}-\varepsilon^{\prime}\right]\left(\alpha_{3}-\alpha_{2} q^{2}\right)-\delta \varepsilon\left(\eta_{1} q^{4}+\eta_{12} q^{2}+\eta_{2}\right)\right\}} .
$$

Let us stress the fact that the behavior of the potential $U_{0}(q)$ is deeply affected by the value of the angular frequency $\omega$ : actually, not only $\mathscr{R}(\omega)$ as defined in (29), but also $\varepsilon^{\prime}=\varepsilon^{\prime}(\omega)$ and $\delta \varepsilon=\delta \varepsilon(\omega)$, according to

$$
\delta \varepsilon=\varepsilon_{\infty}-\varepsilon_{\perp}+\frac{\varepsilon_{0} \varepsilon_{\infty}}{1+\left(\omega / \omega_{D}\right)^{2}}
$$

see the second equation of the system (5). Assuming the reversal point to be close to the Debye frequency $\omega_{i} \cong \omega_{D}$, the condition

$$
\delta \varepsilon(\omega \Rightarrow \infty) \cong-\delta \varepsilon(\omega=0)
$$

has to be satisfied, providing $\omega_{\infty}, \delta \varepsilon(\omega)$ from the data $\varepsilon_{0}^{\prime} \equiv \varepsilon^{\prime}(\omega=0)$, and $\varepsilon_{\perp} \cong$ const. The minimum of the field $E_{0}(q)$, or of the potential $U_{0}(q)$, provides the threshold condition

$$
\begin{aligned}
& E_{\mathrm{th}}=\min _{q \geqslant 0} E_{0}(q), \\
& U_{\mathrm{th}}=\min _{q \geqslant 0} U_{0}(q) .
\end{aligned}
$$

In particular, close to the inversion point, but when $\omega \leqslant \omega_{i}$, where it always is $\delta \varepsilon \geqslant 0$, the usual aperiodic Fréedericksz threshold is recovered as

$$
U_{a}=\pi\left(\frac{K_{11}}{\varepsilon_{0} \delta \varepsilon}\right)^{1 / 2}
$$

which turns out to be real and finite only for $\delta \varepsilon>0$, as expected. Instead, in the hypothesis $\delta \sigma=0$ from Eq. (28), assuming $\sigma /\left(\omega \varepsilon^{\prime \prime} \varepsilon_{0}\right) \ll 1$, the field $E_{0}$ is given by

$$
E_{0}^{2} \cong \frac{2 K \bar{\eta} q_{z}^{2}\left(q^{2}+1\right)^{2}}{\left(\alpha_{3}-\alpha_{2} q^{2}\right) \varepsilon_{\perp} \varepsilon_{0}} .
$$

Thus, supposing $\left|\alpha_{2}\right| \gg\left|\alpha_{3}\right|, \gamma_{1} \sim-\alpha_{2}$, the threshold field for the generalized periodic Fréedericksz transition is obtained by minimizing, as

$$
E_{p}^{2}=\frac{8 K \bar{\eta} q_{z}^{2}}{\gamma_{1} \varepsilon_{\perp} \varepsilon_{0}}
$$

the critical reduced wave vector being

$$
q_{c}=1 .
$$

Moreover, as is known from theory, in the case of strong anchoring the boundary conditions provide the out-of-plane wave vector as

$$
q_{z} \cong \frac{\pi}{d}
$$

which actually corresponds to the smallest possible director deformation across a NLC layer with thickness $d$. Thus we conclude that in the inversion point the domains arise as elongated in the direction perpendicular to the initial planar orientation at the threshold voltage: 


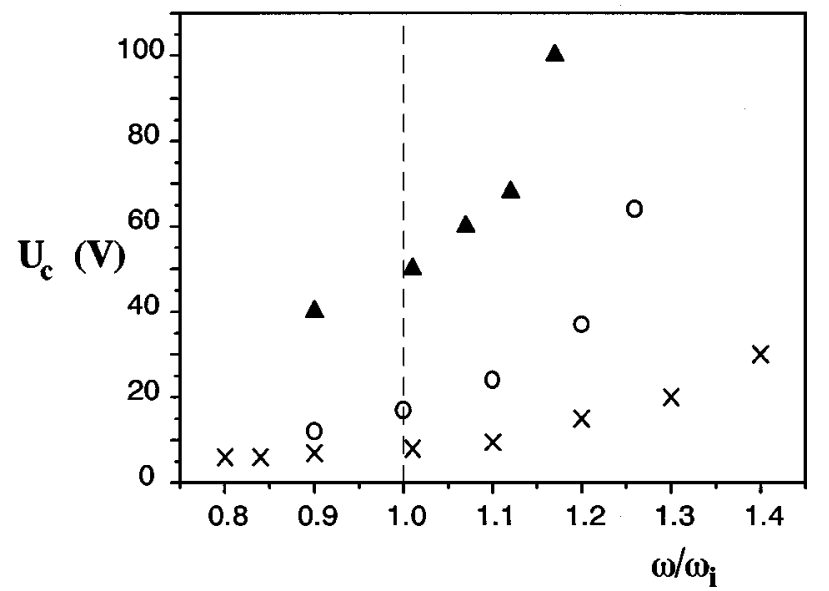

FIG. 3. Critical electric potential $U_{c}$ for periodic steady deformation as a function of the reduced angular frequency $\omega / \omega_{i}$. Experimental data for three different NLC mixtures with $\delta \varepsilon_{0}=4.7(\times$ data), $\delta \varepsilon_{0}=0.35$ ( $\bigcirc$ data), and $\delta \varepsilon_{0}=0.05$ ( $\triangle$ data), where $\delta \varepsilon_{0} \equiv \delta \varepsilon(\omega=0)$ practically is the real dielectric anisotropy at very low frequency.

$$
U_{p}=E_{p} d=4 \pi\left[\frac{K \bar{\eta}}{2 \gamma_{1} \varepsilon_{\perp} \varepsilon_{0}}\right]^{1 / 2},
$$

with a critical wavelength given by

$$
\lambda_{c}=\frac{2 \pi}{q_{c} q_{z}}=2 d .
$$

We stress the fact that in both Eqs. (36) and (39) the threshold value for the periodic texture depends neither on the sign of $\delta \varepsilon$ nor on its absolute value: the periodic distortion can arise also for $\delta \varepsilon \leqslant 0$. The experimental observations for several NLC mixtures [6], reported in Figs. 3 and 4, show good agreement with the predictions given by Eq. (40) for the critical wavelength $\lambda_{c}$ at the threshold.

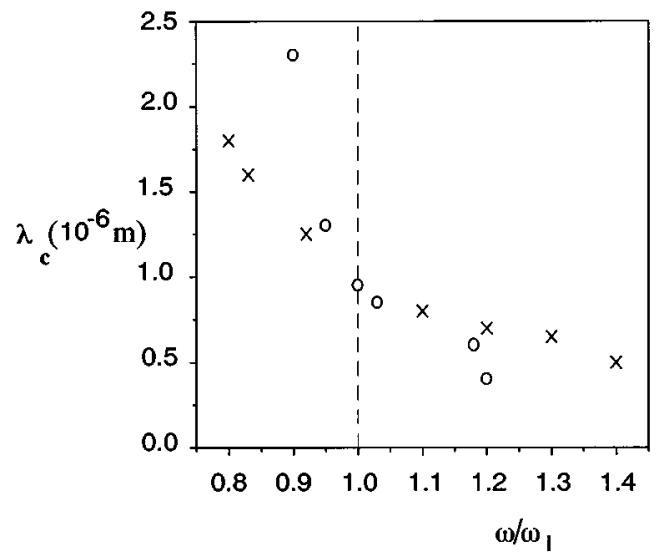

FIG. 4. Critical wavelength $\lambda_{c}$ of the splay-bend periodic distortion, as a function of the reduced angular frequency $\omega / \omega_{i}$. Experimental data for two different NLC mixtures with $\delta \varepsilon_{0}=4.7(\times$ data) and $\delta \varepsilon_{0}=0.35$ ( $\bigcirc$ data).

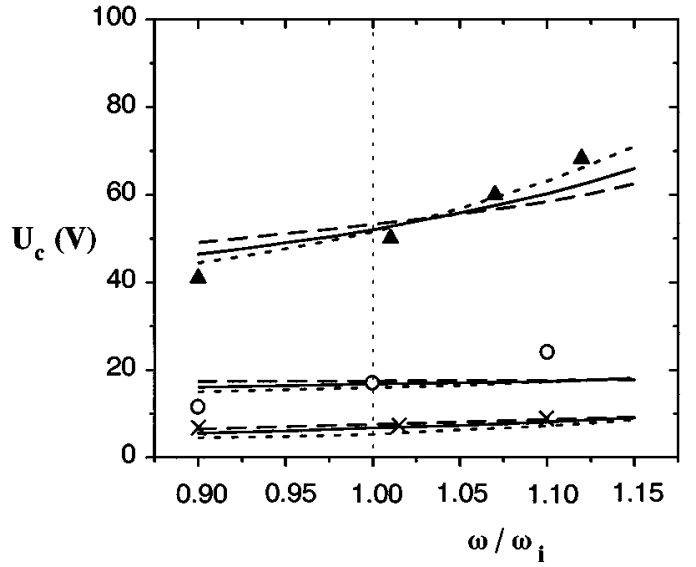

FIG. 5. Best fit (continuous line) of the experimental data for the three NLC mixtures of Fig. 3, close to the dielectric sign reversal point $\omega_{i}$. The best choice of the leading parameters $\alpha_{2}, \alpha_{5}$, and $2 \omega \varepsilon_{0} /\left(\sigma_{\perp}\right)$ is $-0.02 \mathrm{~kg} / \mathrm{m} \mathrm{s}, 0.20 \mathrm{~kg} / \mathrm{m} \mathrm{s}$, and 10 , respectively. The dotted line corresponds to $2 \omega \varepsilon_{0} /\left(\sigma_{\perp}\right)=5$ and the dashed one to $2 \omega \varepsilon_{0} /\left(\sigma_{\perp}\right)=20$, with the same values as previously estimated for $\alpha_{2}$ and $\alpha_{5}$

\section{DISCUSSION AND CONCLUSION}

To discuss the agreement between the critical voltage for the generalized periodic Fréedericksz transition predicted by our model and the one obtained by the experimental observations [6], it is first necessary to give an estimate of the viscosity and conductivity parameters that are included in the present theory; see Eq. (30). First of all, the inversion angular frequency is supposed to be high enough to be comparable with the Debye frequency, so that $\omega_{i} \cong \omega_{D}$ : the experimental observations confirm this statement. Another parameter appearing in the theory is the real part of the ratio between the parallel and the perpendicular conductivity $\sigma^{\prime} / \sigma_{\perp}$. As shown by the experiments, this parameter is unessential when moving in the range 1-3: this is also verified by the numerical analysis of the critical potential, as predicted by the present theory; see Eq. (30). We assume, for the sake of simplicity, that $\sigma^{\prime} / \sigma_{\perp}=2.17$, as for one of the mesogenic mixtures used in the experiments.

Concerning the viscosity parameters, $\alpha_{1}=5 \times 10^{-3} \mathrm{~kg} / \mathrm{m} \mathrm{s}$, $\alpha_{3}=-1 \times 10^{-3} \mathrm{~kg} / \mathrm{m} \mathrm{s}$, and $\alpha_{4}=50 \times 10^{-3} \mathrm{~kg} / \mathrm{m} \mathrm{s}$ are assumed as standard values. The elastic constants $K_{11}$ and $K_{33}$ are considered to be equal, i.e., $K_{11} \sim K_{33} \sim 1 \times 10^{-11} \mathrm{~N}$. The viscous coefficients $\alpha_{2}$ and $\alpha_{5}$ turn out to be much more critical than the others for achieving the minimum of the function $U(q)$ providing the critical potential, as demonstrated by the numerical simulation: such a parameters have to be estimated by comparing the theoretical threshold $U_{p}$ following Eq. (30) with the experimental values, minimizing the mean square deviation.

The experimental values for several NLC mixtures having different dielectric real anisotropy $\delta \varepsilon=\operatorname{Re}\left(\varepsilon_{a}\right)$ are reported in Figs. 3 and 4. The best choice turns out to be $\alpha_{2}=-20 \times 10^{-3}$ $\mathrm{kg} / \mathrm{m} \mathrm{s}$, whereas $\alpha_{5}$ depends on the value of $\delta \varepsilon$ and ranges from 0.1 and $0.3 \mathrm{~kg} / \mathrm{m} \mathrm{s}$. We stress the fact that these values of the viscosity parameters are consistent with the data available in the literature [16-20].

The preceding comment is devoted to the parameter $\varepsilon_{\perp}$, 
which is assumed to be equal to $\sim 6$, as measured for the NLC compounds used in the experiments. As seen from the experimental data (see Fig. 3), around the dielectric inversion point, for small absolute values of the real dielectric anisotropy, the threshold is strongly dependent on the frequency: from the numerical simulation such an important dependence is demonstrated to appear only if the term $2 \omega \varepsilon_{0} / \sigma_{\perp}$ is comparable with the ratio $\sigma^{\prime} / \sigma_{\perp}$, that is, if $1<2 \omega \varepsilon_{0} / \sigma_{\perp}<20$.

As can be seen from Fig. 5, the best choice turns out to be, according to the minimum square method, $2 \omega \varepsilon_{0} / \sigma_{\perp} \sim 5$ in the case of low dielectric anisotropy $\left(\delta \varepsilon_{0}=0.05\right)$ and $2 \omega \varepsilon_{0} / \sigma_{\perp} \sim 10$, for $\delta \varepsilon_{0}=0.35$ and 4.7 , where $\delta \varepsilon_{0} \equiv \delta \varepsilon(\omega=0)$; see Eq. (31). This result is in agreement with the experimental observations of the parameter $2 \omega_{i} \varepsilon_{0} / \sigma_{\perp}$.

Based on these facts, we come to the conclusion that the quasistatic modulated structure near the dielectric sign rever- sal frequency arises mainly due to the dielectric loss and not only in the frequency region, where the usual aperiodic Fréedericksz transition ceases to exist $(\delta \varepsilon>0)$, but also where the initial $P$ orientation in principle should be stabilized $(\delta \varepsilon<0)$. Both the free ions and the apparent space charge created by the dielectric loss interact essentially with the external field providing a drag force, which causes a steady electrohydrodynamic motion in the plane perpendicular to the domain direction. The NLC director distribution remains steady and the effective space charge is steady as well, oscillating with the same frequency of the applied field and with a convenient phase shift. The observed texture can be called quasistatic since the stripe borders actually are static, also in the presence of a regular bidimensional distribution of the steady velocity field. The quasistatic model proposed here describes well the experimental data.
[1] P. G. de Gennes, The Physics of Liquid Crystals (Clarendon, Oxford, 1974).

[2] W. H. de Jeu, C. J. Gerritsma, P. Van Zanten, and W. J. A. Goossens, Phys. Lett. 39A, 335 (1972).

[3] W. H. de Jeu and Th. W. Lathauwers, Mol. Cryst. Liq. Cryst. 26, 235 (1974).

[4] W. J. A. Goossens, Phys. Lett. 40A, 95 (1972).

[5] P. R. Gerber, J. Phys. (Paris) 46, 1865 (1985).

[6] V. G. Chigrinov, T. V. Korkishko, M. I. Barnik, and A. N. Trufanov, Mol. Cryst. Liq. Cryst. 129, 283 (1985).

[7] L. M. Blinov and V. G. Chigrinov, Electrooptic Effects in Liquid Crystal Materials (Springer-Verlag, New York, 1994).

[8] F. M. Leslie, Advances in Liquid Crystals (Academic, New York, 1979).

[9] A. Sparavigna, L. Komitov, O. D. Lavrentovich, and A. Strigazzi, J. Phys. (France) II 2, 1881 (1992).

[10] A. Sparavigna and A. Strigazzi, Mol. Cryst. Liq. Cryst. 221, 109 (1992).

[11] A. Sparavigna, O. D. Lavrentovich, and A. Strigazzi, Phys. Rev. E 49, 1344 (1994).
[12] S. A. Pikin, Structural Transformations in Liquid Crystals (Gordon and Breach, New York, 1991).

[13] G. Barbero, A. Sparavigna, and A. Strigazzi, Nuovo Cimento D 12, 79 (1990), and references therein.

[14] J. L. Ericksen, Arch. Ration. Mech. Anal. 10, 14 (1962).

[15] S. A. Pikin and V. G. Chigrinov, Zh. Eksp. Teor. Fiz. 78, 246 (1980) [Sov. Phys. JETP 51, 123 (1980)].

[16] G. Gähwiller, Phys. Lett. 36A, 311 (1971); Mol. Cryst. Liq. Cryst. 20, 301 (1973).

[17] M. Meisowicz, Mol. Cryst. Liq. Cryst. 97, 1 (1983).

[18] W. W. Beens and W. H. de Jeu, J. Phys. (Paris) 44, 129 (1983).

[19] H. Kneppe and F. Scheider, Mol. Cryst. Liq. Cryst. 65, 23 (1981).

[20] M. I. Barnik, S. V. Belyaev, M. F. Grebenkin, V. G. Rumyantsev, V. A. Seliverstov, V. A. Tsvetkov, and N. M. Shtykov, Krystallografiya 23, 805 (1978) [Sov. Phys. Crystallogr. 23, 451 (1978)]. 